\title{
How many measurements are needed to provide reliable information in terms of the ambulatory arterial stiffness index? the Ohasama study
}

\author{
Masahiro Kikuya ${ }^{1,2}$, Jan A Staessen ${ }^{2}$, Takayoshi Ohkubo ${ }^{1}$, Lutgarde Thijs ${ }^{2}$, Kei Asayama ${ }^{1}$, Michihiro Satoh ${ }^{1}$, \\ Takanao Hashimoto ${ }^{1}$, Takuo Hirose ${ }^{1}$, Hirohito Metoki ${ }^{1}$, Taku Obara ${ }^{1}$, Ryusuke Inoue ${ }^{1}$, Yan $\mathrm{Li}^{2,3}$, \\ Eamon Dolan ${ }^{4}$, Haruhisa Hoshi ${ }^{5}$, Kazuhito Totsune ${ }^{1}$, Hiroshi Satoh ${ }^{1}$, Ji-Guang Wang ${ }^{3}$, Eoin O’Brien ${ }^{6}$ \\ and Yutaka Imai ${ }^{1}$
}

The aim of this study was to investigate how frequent ambulatory blood pressure (ABP) readings need to be obtained to reproduce the ambulatory arterial stiffness index (AASI) and pulse pressure (PP) without loss of information. We compared concordance from full and reduced ABP recordings. We recorded 24-h ABP at 30-min intervals in 1542 residents of Ohasama, Japan (baseline age, 40-93 years; $63.4 \%$ women). We randomly excluded up to 16 readings per recording or we selected readings at fixed 1- or 2-h intervals. Using full recordings as reference, we computed for the reduced recordings repeatability coefficient by Bland and Altman's approach. By Cox regression, we also calculated multivariate-adjusted hazard ratios for cardiovascular mortality. The median number of ABP readings per recording was 46 . Randomly excluding more readings reduced the concordance of AASI, but not PP. Selecting blood pressure readings at 1- or 2-h intervals produced mean values of AASI and PP, which significantly differed from those in full recordings. During follow-up (median, 13.3 years) 126 cardiovascular deaths occurred. Across quartiles, AASI significantly predicted cardiovascular mortality in a U-shaped manner. AASI lost its prognostic significance when the number of randomly excluded readings increased from 8 to 16 or when the interval between readings was $1 \mathrm{~h}$ or longer. Compared with PP, AASI is less reproducible when the number of readings in ABP decreases, but this does not affect the predictive accuracy of AASI for cardiovascular mortality, until the median number of readings per ABP recording is less than $\sim 35$.

Hypertension Research (2011) 34, 314-318; doi:10.1038/hr.2010.240; published online 2 December 2010

Keywords: ambulatory arterial stiffness index; ambulatory blood pressure monitoring; cardiovascular mortality; prospective population study

\section{INTRODUCTION}

We previously defined the ambulatory arterial stiffness index (AASI) as unity minus the regression slope of diastolic on systolic blood pressure, as measured at the brachial artery by ambulatory monitoring. ${ }^{1,2}$ AASI reflects the dynamic relation between systolic and diastolic blood pressure during the day. Conceptually consistent with a hypothesis put forward in $1914,{ }^{3}$ the stiffer the arterial tree, the closer the regression slope and AASI are to zero and unity, respectively. We validated AASI in terms of established indexes of arterial stiffness. ${ }^{1}$ Several studies ${ }^{2,4-8}$ demonstrated significant association of AASI with signs of target organ damage $e^{4,7}$ or with the incidence of cardiovascular mortality ${ }^{8}$ and morbidity ${ }^{2,5,6}$ in hypertensive patients, ${ }^{7,8}$ or in population samples randomly recruited in Western Europe ${ }^{5}$ and Japan. ${ }^{6}$

In the aforementioned studies, ${ }^{1,2,4-8}$ the interval between programmed blood pressure readings ranged from 15 (see refs 4, 5, 7 and 8) to 30 (see refs 2 and 6) minutes during daytime and from 20 (see ref 7) to 45 (see ref 1) minutes during nighttime. None of the studies addressed the issue how many blood pressure readings are required to calculate in a reproducible manner AASI from 24-h ambulatory readings. To answer this question, we analyzed the Ohasama database ${ }^{9}$ randomly excluding a stepwise increasing number of blood pressure readings from each individual ambulatory blood pressure $(\mathrm{ABP})$ recording according to the approach originally

\footnotetext{
${ }^{1}$ Tohoku University Graduate School of Medicine and Pharmaceutical Sciences, Sendai, Japan; ${ }^{2}$ Studies Coordinating Centre, Division of Hypertension and Cardiovascular Rehabilitation, Department of Cardiovascular Diseases, University of Leuven, Leuven, Belgium; ${ }^{3}$ Shanghai Institute of Hypertension, Shanghai Jiaotong University Medical School, Shanghai, China; ${ }^{4}$ Cambridge University Hospitals, National Health Service Foundation Trust, Addenbrook's Hospital, Cambridge, UK; ${ }^{5}$ Ohasama Hospital, Ohasama, Japan and ${ }^{6}$ Conway Institute of Biomolecular and Biomedical Research, University College Dublin, Dublin, Ireland

Correspondence: Dr M Kikuya, Department of Planning for Drug Development and Clinical Evaluation, Tohoku University Graduate School of Pharmaceutical Science, 6-3 Aramaki-aza-aoba, Sendai 980-8578, Japan.

E-mail: kikuyam@m.tains.tohoku.ac.jp
}

Received 13 July 2010; revised 16 August 2010; accepted 2 September 2010; published online 2 December 2010 
described by Di Rienzo et al. ${ }^{10}$ We also addressed the prognostic accuracy with full and reduced recordings.

\section{METHODS}

\section{Study population}

The Institutional Review Boards of Tohoku University School of Medicine and the Department of Health of the Ohasama Municipal Government approved the study. All participants gave informed written consent. From 1988 until 1994, we contacted all 2716 subjects aged 40 years or more and living in three of four Ohasama districts. Subjects who were not at home during the normal working hours of the study nurses $(n=575)$ and those hospitalized $(n=121)$ or incapacitated $(n=31)$ were not eligible. ${ }^{11}$ Of the remaining 1989 residents, 1552 (78.0\%) participated in the baseline examinations and were followed up. We excluded 10 subjects from this analysis because their ABP recording included less than 8 or $4 \mathrm{~h}$ during the awake and asleep periods, respectively. Thus, the number of participants statistically analyzed totaled $1542.6,1$

\section{ABP measurement}

We programmed validated ABPM-630 recorders (Nippon Colin, Komaki, Japan $^{12}$ to obtain oscillometric blood pressure reading at 30-min intervals throughout the whole day. On monitoring days, participants noted the hour of awakening and going to sleep. According to the diary, 'daytime' and 'nighttime' were determined as the awakening and sleeping periods of the subject, respectively. In agreement with current guidelines, ${ }^{13}$ we defined ambulatory hypertension as an awake blood pressure of at least $135 \mathrm{~mm} \mathrm{Hg}$ systolic or $85 \mathrm{~mm} \mathrm{Hg}$ diastolic, or use of antihypertensive drugs. From 24-h recordings edited according to previously published criteria, ${ }^{14}$ we computed for each participant the regression slope of diastolic on systolic blood pressure. ${ }^{1,2}$ We did not force the regression line through the origin (intercept $=0$ ) because during diastole when flow drops to zero, such phenomenon does not occur for blood pressure $^{15}$ We defined AASI as one minus the regression slope. In addition, from individual ABP recordings and from the same readings as used for AASI, we computed pulse pressure (PP) as the difference between the 24-h systolic and diastolic blood pressure and mean arterial pressure as 24 -h diastolic blood pressure plus one-third of the $\mathrm{PP}$

We first computed AASI and PP, using all available blood pressure readings in each individual recording. Next, we recalculated AASI and PP for each subject randomly excluding from 2 to 16 ABP readings. Finally, we also computed AASI and PP only using ABP readings at fixed 1- or 2-h intervals. Dipping was a night-to-day ratio of systolic blood pressure less than $0.90 .{ }^{16}$

\section{Other measurements}

At baseline, trained nurses measured anthropometric characteristics. Body mass index was weight in kilograms divided by height in meters squared. The study nurses administered a standardized questionnaire, inquiring into each subject's medical history, intake of medications, and smoking and drinking habits.

We followed cardiovascular mortality (ICD-10 codes 'I') until 31 December 2004. ${ }^{6}$ We ascertained vital status from the Ohasama residents' registration cards, which are the basis for the distribution of social security benefits. We obtained the causes of death from the National Japanese Mortality Registry. We verified the diagnoses reported on the death certificates against the medical records of Ohasama hospital, where over $90 \%$ of the Ohasama inhabitants undergo regular health check-ups.

\section{Statistical analysis}

For statistical analysis, we used SAS software, version 9.1 (SAS Institute, Cary, NC, USA). We compared means and proportions by the standard normal $z$-test for large samples and the $\chi^{2}$-statistic, respectively.

We randomly reduced the number of recordings in two reiterating steps. First, we randomly excluded two reading from each recording, using the SURVEYSELECT procedure, as implemented in the SAS package. Next, we computed each participant's AASI and PP from the reduced recording. To exclude more readings in a random manner, we repeated the two steps, always starting from the full recording, but using different seeds in the SURVEYSELECT procedure. Thus, we computed AASI and PP always from the same set of ambulatory readings. The exclusion of a larger number of readings was truly random and not cumulative to the reduction in recordings with fewer readings excluded.

We determined agreement between the ambulatory parameters from original and reduced recordings, using Bland and Altman's ${ }^{17}$ approach and Pearson's correlation coefficients. The repeatability coefficient (RC) was defined as twice the standard deviation (s.d.) of the within-subject differences between the parameters from original and reduced recordings. ${ }^{17}$ The relative RC was RC expressed as a percentage of the maximal variability of the parameter under study. ${ }^{18}$ Maximal variability was four times the s.d. for the between-subjects variation in AASI or PP in the full recordings. RC allows comparing concordance across different definitions of AASI and PP. Higher values of RC and RCC indicate worse concordance.

We also evaluated the consistency of AASI and PP, as determined from original and reduced recordings in the prediction of cardiovascular mortality. We calculated hazard ratios by multiple Cox regression comparing the risk in each quartile of AASI or PP with the overall risk in the whole cohort. We used the deviation from mean coding ${ }^{19}$ because, as previously described, ${ }^{6}$ the association of cardiovascular mortality with AASI and PP was curvilinear. This approach also allows computation of confidence intervals for the hazard ratio in each quartile without definition of an arbitrary reference group. We adjusted the hazard ratios for baseline characteristics, including sex, age, mean arterial pressure, body mass index, current and past smoking, and drinking habits, diabetes mellitus, and a history of cardiovascular disease.

\section{RESULTS}

\section{Baseline characteristics of the subjects}

The 1542 participants included 977 (63.4\%) women and 760 (49.3\%) patients with ambulatory hypertension, of whom $473(62.2 \%)$ were taking antihypertensive drugs. Mean age ( \pm s.d.) was $61.7 \pm 10.7$ years. AASI averaged $0.46 \pm 0.10$ units and PP averaged $51.3 \pm 7.4 \mathrm{~mm} \mathrm{Hg}$. The 24-h blood pressure was $123.2 \pm 13.0 \mathrm{mmHg}$ systolic, and $72.0 \pm 7.7 \mathrm{~mm} \mathrm{Hg}$ diastolic. Height averaged $151.9 \pm 8.8 \mathrm{~cm}$, weight $54.6 \pm 9.2 \mathrm{~kg}$ and body mass index $23.6 \pm 3.1 \mathrm{~kg} \mathrm{~m}^{-2}$. At enrollment, 319 men (56.6\%) were current smokers and 251 (62.4\%) reported intake of alcohol. Among women these numbers were $14(1.4 \%)$ and $28(3.3 \%)$, respectively. The average level of serum total cholesterol was $4.99 \pm 0.88 \mathrm{mmol}^{-1}$. Of the study participants, 268 had diabetes mellitus (17.4\%) and 98 had history of previous cardiovascular disease (6.4\%).

\section{Agreement of AASI and PP by reducing the number of readings} The number of readings available per recording for the calculation of AASI and PP averaged 44.6. The 95th, 75th, 50th, 25th, 10th, 5th and first percentile values were $52,48,46,42,38,36$ and 30 , respectively.

Random exclusion of 2-16 readings from the ambulatory recordings did not consistently change the average value of AASI (Table 1), but increased RC and RCC from 0.040 to 0.113 and from 10.2 to $29.3 \%$, respectively, indicating worsening concordance with more readings omitted from analysis. The corresponding correlation coefficients expressing concordance of AASI between the full, that is, one reading every $30 \mathrm{~min}$ and randomly reduced recordings range dropped from 0.98 to 0.87 . The fixedly reduced recordings produced average values of AASI, which significantly differed from those in the full recordings. Fixedly spacing the interval between readings by 1 and $2 \mathrm{~h}$ increased RC and RCC to 0.119 and 0.213 , and to 30.5 and $54.7 \%$, respectively. The corresponding Pearson's correlation coefficients were 0.85 and 0.65 .

Random exclusion of readings from ambulatory recordings did not significantly change the average value of PP (Table 2), whereas the fixedly reduced recordings produced average PP values, which were significantly higher than those in the full recordings were. However, for PP, both the random and fixed exclusion of readings had little influence on RC, RCC and the correlation coefficients. 
Table 1 Agreement of AASI between full ${ }^{\mathrm{a}}$ and reduced recordings

\begin{tabular}{lccccc}
\hline Readings & $\begin{array}{c}\text { AASI } \\
\text { (units) }\end{array}$ & $\begin{array}{c}\Delta \pm \text { s.d. } \\
\text { (units) }\end{array}$ & $\begin{array}{c}R C \\
\text { (units) }\end{array}$ & $\begin{array}{c}R R C \\
\text { (\%) }\end{array}$ & $r$ \\
\hline All readings $^{\mathrm{a}}$ & $0.457 \pm 0.097$ & & & & \\
\multicolumn{7}{c}{ Random exclusion of readings } & & & & & \\
2 readings & $0.456 \pm 0.098$ & $-0.0013 \pm 0.0199$ & 0.04 & 10.2 & 0.98 \\
4 readings & $0.457 \pm 0.100$ & $-0.0003 \pm 0.0217$ & 0.04 & 11.2 & 0.98 \\
8 readings & $0.456 \pm 0.103$ & $-0.0006 \pm 0.0330$ & 0.07 & 17.0 & 0.95 \\
16 readings & $0.459 \pm 0.115$ & $0.0018 \pm 0.0564$ & 0.11 & 29.0 & 0.87 \\
& & & & & \\
$\begin{array}{l}\text { Non-random selection of readings } \\
\text { One reading } \\
\text { per hour }\end{array}$ & $0.454 \pm 0.113$ & $-0.0035 \pm 0.0593$ & 0.12 & 30.5 & 0.85 \\
$\begin{array}{l}\text { One reading } \\
\text { per 2 } \mathrm{h}\end{array}$ & $0.447 \pm 0.139$ & $-0.0101 \pm 0.1065$ & 0.21 & 54.7 & 0.65 \\
\hline
\end{tabular}

Abbreviations: AASI, ambulatory arterial stiffness index; RC, repeatability coefficient; RRC, relative repeatability coefficient.

${ }^{a} O$ ne reading per $30 \mathrm{~min}$. Plus-minus values are mean \pm s.d. Mean difference $(\Delta)$ was computed as AASI from reduced recording minus AASI from full recordings. RC, RRC and $r$ indicate the

repeatability coefficient, the relative repeatability coefficient and Pearson's correlation

coefficient. For further clarification, see Methods and reference.

Table 2 Agreement of 24-h Pulse Pressure (PP) between full ${ }^{a}$ and reduced recordings

\begin{tabular}{lccccc}
\hline & $P P$ & $\Delta \pm s . d$. & $R C$ & $R R C$ & \\
Readings & $(\mathrm{mm} \mathrm{Hg})$ & $(\mathrm{mm} \mathrm{Hg})$ & $(\mathrm{mm} \mathrm{Hg})$ & $(\%)$ & $\mathrm{r}$ \\
\hline
\end{tabular}

All readings $^{\mathrm{a}} \quad 51.29 \pm 7.41$

\begin{tabular}{lrrrrr}
\multicolumn{2}{l}{ Random exclusion of readings } & & & & \\
2 readings & $51.27 \pm 7.41$ & $-0.0143 \pm 0.3719$ & 0.74 & 2.5 & 1.00 \\
4 readings & $51.28 \pm 7.42$ & $-0.0095 \pm 0.5010$ & 1.00 & 3.4 & 1.00 \\
8 readings & $51.28 \pm 7.43$ & $-0.0073 \pm 0.7556$ & 1.51 & 5.1 & 0.99 \\
16 readings & $51.35 \pm 7.51$ & $0.0589 \pm 1.2021$ & 2.40 & 8.1 & 0.99
\end{tabular}

Non-random selection of readings

\begin{tabular}{lccccc}
$\begin{array}{l}\text { One reading } \\
\text { per hour }\end{array}$ & $51.41 \pm 7.49$ & $0.1194 \pm 1.2161$ & 2.43 & 8.2 & 0.99 \\
$\begin{array}{l}\text { One reading } \\
\text { per } 2 \mathrm{~h}\end{array}$ & $51.72 \pm 7.74$ & $0.4267 \pm 2.0848$ & 4.17 & 14.1 & 0.96 \\
\hline
\end{tabular}

Abbreviations: RC, repeatability coefficient; RRC, relative repeatability coefficient.

aOne reading per $30 \mathrm{~min}$. Plus-minus values are mean $\pm \mathrm{s}$.d. Mean difference $(\Delta)$ was computed as PP from reduced recording minus PP from full recordings. RC, RRC and $r$ indicate the as PP from reduced recording minus PP from full recordings. RC, RRC and $r$ indicate the coefficient. For further clarification, see Methods and reference.

\section{Analysis of cardiovascular mortality}

During follow-up (median, 13.3 years; 5-95th percentile interval, 4.7-16.3 years), 19024 person-years accrued. Of 345 deaths, $126(36.5 \%)$ were cardiovascular, including 63 strokes and 59 cardiac deaths.

Using full recordings, that is, one reading every $30 \mathrm{~min}$, the multivariate-adjusted hazard ratios for cardiovascular death significantly deviated from those in the whole population in an U-shaped manner for AASI (Table 3), whereas for PP none of the hazard ratios significantly departed from the overall risk (Table 4), after adjustment for 24-h mean arterial pressure and other covariates. Next, we computed hazards ratios from reduced recordings. When we randomly excluded readings, the multivariate-adjusted hazard ratios of cardiovascular mortality with AASI lost significance, when the number of excluded readings increased from 8 to 16 (Table 3) or when the interval between readings was $1 \mathrm{~h}$ or longer.

\section{Sensitivity analyses}

In sensitivity analyses, using all available readings, we evaluated whether dipping status and antihypertensive treatment affected estimates of AASI and PP. Both AASI $(0.493 \pm 0.107$ vs. $0.438 \pm 0.086$ units) and PP $(52.8 \pm 8.1$ vs. $50.5 \pm 6.9 \mathrm{~mm} \mathrm{Hg})$ were slightly but significantly $(P<0.0001)$ higher in 526 non-dippers compared with 1016 dippers. AASI was similar in 473 treated patients and 1069 untreated subjects $(0.463 \pm 0.095$ vs. $0.454 \pm 0.098 ; P=0.12)$, whereas $\mathrm{PP}$ was significantly higher in the former group $(54.0 \pm .8 .2 \mathrm{vs}$. $50.1 \pm 7.3 \mathrm{~mm} \mathrm{Hg} ; P<0.0001)$. However, estimates of RC and RCC, as reported in Tables 1 and 2 for AASI and PP, respectively, were not materially different between dippers and non-dippers or between treated and untreated patients (data not shown).

\section{DISCUSSION}

We studied the concordance of AASI and PP, whereas excluding a stepwise increasing number of blood pressure readings from ABP recordings, according to the approach originally described by Di Rienzo et al. ${ }^{10}$ In addition, we evaluated to what extent AASI from full and reduced recordings predicted cardiovascular mortality. In middle-aged and older subjects recruited from a Japanese population, AASI, a measure of the dynamic relation between diastolic and systolic blood pressure throughout the day, ${ }^{1,2}$ could be reproducibly computed from ambulatory recordings with readings programmed at 30-min intervals, until more than eight readings were randomly omitted. Similarly, AASI remained a significant predictor of cardiovascular mortality until more than eight readings were randomly excluded. On the other hand, AASI computed from recordings with the interval between readings set at 1 or $2 \mathrm{~h}$ lost its prognostic value. Estimates of mean PP were also significantly affected by the random exclusion of more than eight readings or by using an interval between readings of 1 or $2 \mathrm{~h}$. However, relative RC of PP in the reduced compared with the full recordings never increased above $15 \%$, whereas for AASI RCC already exceeded the 15\% threshold after the random exclusion of eight readings.

To our knowledge, there are currently no published data on the number of measurements required for AASI. When comparing our current results for PP with the literature, one should realize that as Di Rienzo et al., ${ }^{10}$ we evaluated concordance from reduced $v s$. full recordings, which is different from assessing concordance from repeat (test-retest) measurements. Di Rienzo et al. recorded blood pressure intra-arterially in 20 hypertensive patients and compared estimates from continuous beat-to-beat recordings with those from intermittent measurements at interval. When the interval between the intermittent measurement was $30 \mathrm{~min}$ or shorter, estimates of the 24 -h systolic and diastolic blood pressure means were within $1 \mathrm{~mm} \mathrm{Hg}$ of those based on beat-to-beat recordings. ${ }^{10}$ At 1-h intervals, the 24-h systolic and diastolic differences were $2-3 \mathrm{~mm} \mathrm{Hg} .{ }^{10}$ Di Rienzo et al. ${ }^{10}$ did not report on PP, but from the results on systolic and diastolic pressure one may infer that up to 30-min intervals estimates of 24-h PP would be within $2 \mathrm{~mm} \mathrm{Hg}$ of those from continuous recordings, a result in-line with our current findings. Thijs et al. ${ }^{18}$ studied 97 normotensive volunteers, aged 23-84 years, who underwent ambulatory monitoring with readings programmed at 7.5 -min intervals from 0800 to $2000 \mathrm{~h}$ and at 15 -min intervals for the remainder of the day. If only the readings at 30 -min or $1-\mathrm{h}$ intervals were considered, estimates of PP were within $0.5 \mathrm{~mm} \mathrm{Hg}$ of those derived from the full recordings. 
Table 3 Adjusted Hazard Ratios for cardiovascular mortality by quartiles of AASI derived From fulla and reduced recordings

\begin{tabular}{lccc}
\hline & \multicolumn{2}{c}{ AASI quartile limits, unit } \\
\cline { 2 - 4 } Readings & $<0.38$ & $0.39-0.45$ & $0.45-0.51$ \\
\hline All readings $^{\mathrm{a}}$ & $1.40(1.01-1.95)^{\dagger}$ & $0.82(0.59-1.15)$ & $0.64(0.46-0.91)^{\dagger}$ \\
Random exclusion of readings & & & \\
$\quad$ readings & $1.35(0.97-1.89)^{\dagger}$ & $0.86(0.62-1.19)$ & $0.68(0.49-0.95)^{\dagger}$ \\
4 readings & $1.30(0.93-1.82)$ & $0.88(0.63-1.24)$ & $0.64(0.45-0.89)^{\dagger}$ \\
8 readings & $1.18(0.84-1.65)$ & $0.88(0.63-1.23)$ & $0.71(0.51-0.99)^{\dagger}$ \\
16 readings & $1.31(0.95-1.82)$ & $0.80(0.56-1.13)$ & $0.84(0.61-1.16)$ \\
Non-random selection of readings & & & $1.35(1.03-1.78)^{\dagger}$ \\
$\quad \begin{array}{l}\text { One reading per hour } \\
\text { One reading per 2 } \mathrm{h}\end{array}$ & $0.99(0.70-1.40)$ & $1.03(0.74-1.42)$ & $0.80(0.58-1.12)$ \\
\hline
\end{tabular}

Abbreviation: AASI, ambulatory arterial stiffness index.

a One reading per $30 \mathrm{~min}$. The hazard ratios ( $95 \%$ confidence interval) express the risk in each quartile vs, the overall risk in the whole study population. All hazard ratios were adjusted for sex, age, body mass index, 24-h mean arterial pressure, past and current smoking and drinking, diabetes mellitus, and a history of cardiovascular disease. Significance of the hazard ratios: ${ }^{\dagger} P<0.05$.

Table 4 Adjusted Hazard Ratios for cardiovascular mortality by quartiles of PP derived from fulla and reduced recordings

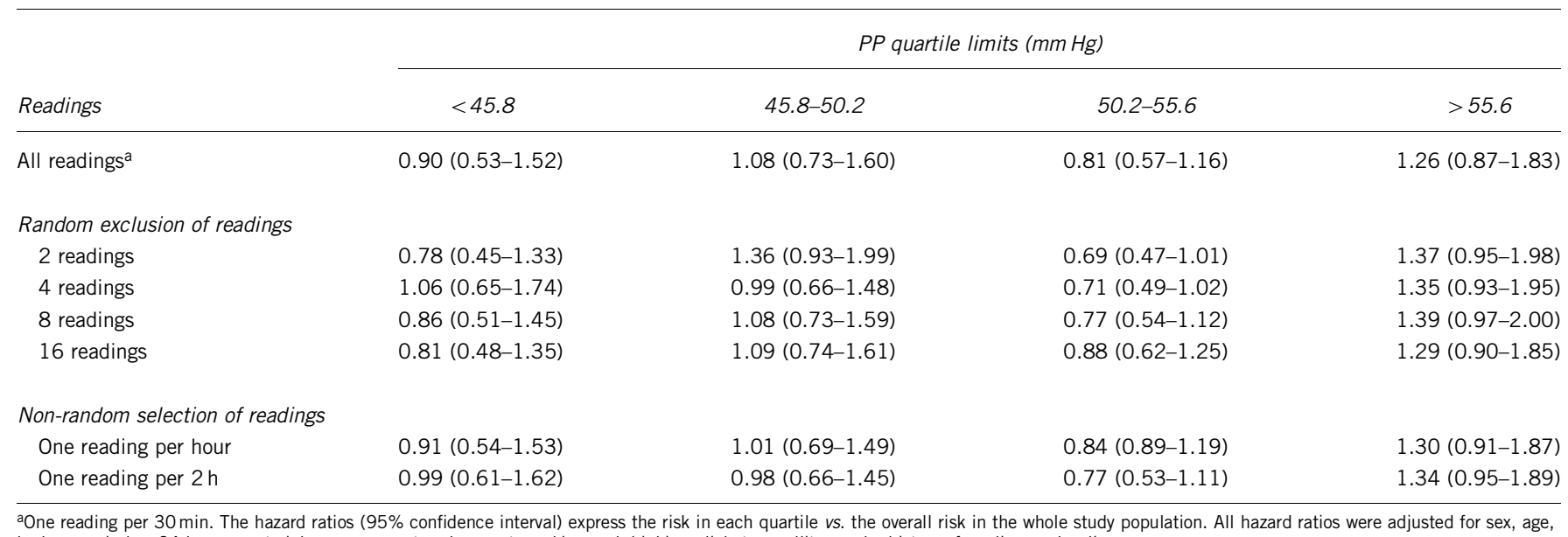

body mass index, 24-h mean arterial pressure, past and current smoking and drinking, diabetes mellitus and a history of cardiovascular disease.

Our current results show that estimates of PP are less vulnerable to an increasing interval between successive ambulatory readings than AASI. This is not surprising. Previous studies clarified that the 24-h systolic and diastolic blood pressures are highly reproducible, even if the number of readings are reduced to one every $30 \mathrm{~min}^{10}$ or to 1 per hour. ${ }^{18}$ In Thijs et al. ${ }^{18}$ study, the relative RC for 24 -h blood pressure in recordings with only 1-hourly readings compared with the full recordings was $9 \%$ systolic and $12 \%$ diastolic. For the night-to-day blood pressure difference, RCC was 23\% systolic and 30\% diastolic. For all variables that involved computation of statistical parameters from whole-day recordings, estimates of RCC for a 1-h interval between readings $v s$. full recordings were consistently higher than $30 \%$ with values up to $80 \%$. PP is the simple difference between the average systolic and diastolic blood pressure over $24 \mathrm{~h}$, which are measurements with high concordance in reduced recordings. In contrast, AASI is a parameter derived by linear regression from individual readings in one person's ambulatory recordings. Individual blood pressure readings in ambulatory recordings are not reproducible.

In this study, up to eight readings could be randomly excluded from ABP recordings with readings programmed at 30-min intervals before AASI started to lose its prognostic significance, whereas PP did not predict cardiovascular death neither in full, nor in reduced recordings. The Dublin Outcome Study involved 11291 patients, who over a median follow-up of 5.3 years experienced 566 cardiovascular deaths, including 151 from stroke and 358 from cardiac disorders. ${ }^{2}$ Before and after adjustment for other cardiovascular risk factors, AASI and PP significantly predicted total cardiovascular mortality. ${ }^{2}$ AASI was a stronger predictor than PP for stroke (mutually adjusted relative hazard ratios for 1 s.d. increase, $1.21(P=0.02)$ vs. 1.04 $(P=0.66))$ with the opposite trend for cardiac mortality (1.03 $(P=0.63)$ vs. $1.21(P=0.002)) .^{2}$ In the Ohasama population stroke represents $50 \%$ of cardiovascular mortality, ${ }^{6}$ whereas in the Irish patients this proportion was $26.7 \%{ }^{2}$ The AASI, not PP, predicted cardiovascular mortality in the Ohasama cohort was therefore in-line with previous findings. ${ }^{2,6}$ Aortic pulse-wave velocity, in the experts' view the gold standard to measure arterial stiffness, ${ }^{20}$ is a strong predictor of outcome over and beyond classical risk factors. We recently assessed the independent contributions of aortic pulse-wave velocity and AASI to the stratification of cardiovascular risk in a Danish cohort sample. ${ }^{21}$ In multivariate-adjusted Cox regression models including both indexes, ${ }^{21}$ AASI (standardized hazard ratio, 1.68; $P=0.01$ ) but not aortic pulse-wave velocity $(0.91 ; P=0.62)$ predicted stroke, whereas the opposite was true for the composite of 
all cardiovascular events (aortic pulse-wave velocity, 1.15; $P=0.03$ and AASI, 1.04; $P=0.68$ ).

This study must be interpreted within the context of its potential limitations. First, we programmed our ABP readings at a constant interval during the day and night for $30 \mathrm{~min}^{2}$ However, from the current results as well as from those published before, ${ }^{10,18}$ it is unlikely that a shorter interval between consecutive measurements in the full recordings, of for instance $15 \mathrm{~min}$, would have had a bearing on the present conclusions. Second, middle-aged and older women made up the majority of the Ohasama cohort. Furthermore, only subjects who were at home during normal working hours were included in the study. This might to some extent limit the generalizability of our findings. On the other hand, the proportion of patients on antihypertensive drug treatment was lower in our population study than in cohorts of hypertensive patients. ${ }^{2}$ Finally, we studied only mortality and we cannot extrapolate our current results to the combination of fatal and non-fatal outcomes.

In conclusion, compared with PP, AASI is less reproducible when the number of readings in $\mathrm{ABP}$ decreases, but this does not affect the predictive accuracy of AASI for cardiovascular mortality, until the median number of readings per ABP recording becomes less than $\sim 35$.

\section{CONFLICT OF INTEREST}

The authors declare no conflict of interest.

\section{ACKNOWLEDGEMENTS}

The authors gratefully acknowledge the clerical assistance of Mrs Youko Kanno, Ms Miwa Suzuki (Sendai, Japan) and Ms Sandra Covens (Leuven, Belgium). The Ministries of Education, Culture, Sports, Science and Technology (grants for scientific research: 15790293, 17790381, 18390192, 18590587, 19590929, 19790423, 20590629, 21390201, 21591016 and 22590767) and of Health, Labor and Welfare (health science research grants and medical technology evaluation grants), Grant-in-Aid for Japan Society for the Promotion of Science (JSPS) fellows (16.54041, 18.54042, 19.7152, 20.7198, 20.7477 and 20.54043), the Japan Atherosclerosis Prevention Fund, the Uehara Memorial Foundation, and the Takeda Medical Research Foundation, National Cardiovascular Research Grants; and Biomedical Innovation Grants supported research in Japan. The Flemish Fund for Scientific Research, Brussels (grant G.0453.05) and the University of Leuven (grant OT/05/49) gave support to the Studies Coordinating Centre in Leuven. The Bilateral Scientific and Technological Collaboration between China and Flanders, Ministry of the Flemish Community, Brussels (grant BIL02/10) supported the postdoctoral research of Yan Li in Leuven, Belgium.

1 Li Y, Wang JG, Dolan E, Gao PJ, Guo HF, Nawrot T, Stanton AV, Zhu DL, O'Brien E, Staessen JA. Ambulatory arterial stiffness index derived from 24-h ambulatory blood pressure monitoring. Hypertension 2006; 47: 359-364.
2 Dolan E, Thijs L, Li Y, Atkins N, McCormack P, McClory S, O'Brien E, Staessen JA, Stanton AV. Ambulatory arterial stiffness index as a predictor of cardiovascular mortality in the Dublin Outcome Study. Hypertension 2006; 47: 365-370.

3 MacWilliam JA, Melvin GS. Systolic and diastolic blood pressure estimation with special reference to the auditory method. Br Med J 1914; 28: 693-697.

4 Leoncini G, Ratto E, Viazzi F, Vaccaro V, Parodi A, Falqui V, Conti N, Tomolillo C, Deferrari G, Pontremoli R. Increased arterial stiffness index is associated with target organ damage in primary hypertension. Hypertension 2006; 48: 397-403.

5 Hansen TW, Staessen JA, Torp-Pedersen C, Rasmussen S, Li Y, Dolan E, Thijs L, Wang JG, O'Brien E, Ibsen H, Jeppesen J. Ambulatory arterial stiffness index predicts stroke in a general population. J Hypertens 2006; 24: 2247-2253.

6 Kikuya M, Staessen JA, Ohkubo T, Thijs L, Metoki H, Asayama K, Obara T, Inoue R, Li Y, Dolan E, Hoshi H, Hashimoto J, Totsune K, Satoh H, Wang JG, O'Brien E, Imai Y. Ambulatory arterial stiffness index and 24-h ambulatory pulse pressure as predictors of mortality in Ohasama, Japan. Stroke 2007; 38: 1161-1166.

7 Mule G, Cottone S, Cusimano P, Incalcaterra F, Giandalia M, Costanzo M, Nardi E, Palermo A, Geraci C, Costa R, Cerasola G. Inverse relationship between ambulatory arterial stiffness index and glomerular filtration rate in arterial hypertension. Am J Hypertens 2008; 21: 35-40.

8 Muxfeldt ES, Cardoso CR, Dias VB, Nascimento AC, Salles GF. Prognostic impact of the ambulatory arterial stiffness index in resistant hypertension. J Hypertens 2010; 28: 1547-1553.

9 Ohkubo T. Prognostic significance of variability in ambulatory and home blood pressure from the Ohasama study. J Epidemiol 2007; 17: 109-113.

10 Di Rienzo M, Grassi G, Pedotti A, Mancia G. Continuous vs intermittent blood pressure measurements in estimating 24-h average blood pressure. Hypertension 1983; 5: 264-269.

11 Ohkubo T, Imai Y, Tsuji I, Nagai K, Ito S, Satoh H, Hisamichi S. Reference values for 24-h ambulatory blood pressure monitoring based on a prognositic criterion. The Ohasama Study. Hypertension 1998; 32: 255-259.

12 Imai Y, Abe K, Sasaki S, Minami N, Munakata M, Sekino H, Nihei M, Yoshinaga K. Determination of clinical accuracy and nocturnal blood pressure pattern by new portable device for monitoring indirect ambulatory blood pressure. Am J Hypertens 1990; 3: 293-301.

13 European Society of Hypertension/European Society of Cardiology Guidelines Committee. 2003 European society of hypertension/European society of cardiology guidelines for the management of arterial hypertension. J Hypertens 2003; 21 : 1011-1053.

14 Imai Y, Nihei M, Abe K, Sasaki S, Minami N, Munakata M, Yumita S, Onoda H, Sekino H, Yamakoshi K, Yoshinaga K. A finger volume-oscillometric device for monitoring ambulatory blood pressure: laboratory and clinical evaluation. Clinical and Experimental Hypertension-Theory and Practice 1987; 9: 2001-2025.

15 Nichols WW, O'Rourke M. McDonald's blood flow in arteries. Therorectical, experimental and clinical principles. In: Arnold E (ed). London, Sydney, Auckland, 1998, pp 54-401.

16 Boggia J, Li Y, Thijs L, Hansen TW, Kikuya M, Bjrklund-Bodegrd K, Richart T, Ohkubo T, Kuznetsova T, Torp-Pedersen C, Lind L, Ibsen H, Imai Y, Wang JG, Sandoya E, O'Brien E, Staessen JA, International Database on Ambulatory blood pressure monitoring in relation to Cardiovascular Outcomes (IDACO) investigators. Prognostic accuracy of day versus night ambulatory blood pressure: a cohort study. Lancet 2007; 370: 1219-1229.

17 Bland JM, Altman DG. Statistical methods for assessing agreement between two methods of clinical measurement. Lancet 1986; 2: 307-310.

18 Thijs L, Staessen J, Fagard R, Zachariah P, Amery A. Number of measurements required for the analysis of diurnal blood pressure profile. J Hum Hypertens 1994; 8: 239-244.

19 Hosmer DJ, Leleshow S. Applied Logistic Regression. John Wiley \& Sons: New York, 1989, pp 47-56.

20 Laurent S. Surrugate measures of arterial stiffness: do they have additive predictive value or are they only surrogates of a surrogate? Hypertension 2006; 47: 325-326.

21 Hansen TW, Li Y, Staessen JA, Jeppesen J, Rasmussen S, Wang JG, Thijs L, Ibsen H, Safar ME, Torp-Pedersen C. Independent prognostic value of the ambulatory arterial stiffness index and aortic pulse wave velocity in a general population. $J$ Hum Hypertens 2008; 22: 214-216 